\title{
ENSAYOS DE DEGRADACIÓN A CAMPO DE ALAMO CRIOLLO (Populus nigra cv Italica) PRESERVADO, ESTUDIO DE ALGUNAS PROPIEDADES
}

\author{
Keil, Gabriel ${ }^{1}$, Nosetti, Julia² ${ }^{2}$ Spavento, Eleana ${ }^{3}$, Monelos, Lucas ${ }^{4}$ y Días, Boris ${ }^{5}$
}

\section{RESUMEN}

En la región patagónica en general y en la provincia de Santa Cruz en particular, existe un recurso importante de álamo criollo, Populus nigra cv Italica, concentrado principalmente en cortinas protectoras de cultivos fruti-hortícolas, el cual podría constituir un importante recurso maderero regional.

Dentro de las virtudes de la madera de esta especie, se encuentran su color claro, su grano derecho, la ausencia de extractivos y si bien su durabilidad natural es baja y limita su uso en condiciones de riesgo severas, presenta la característica de ser fácilmente impregnable.

Los ensayos de degradación a campo permiten evaluar la durabilidad natural o adquirida de una madera ante exposiciones a distintas condiciones de riesgo en servicio. Más allá de la evaluación tradicional de un ensayo de degradación a campo, a través de la pérdida de peso de la madera en condiciones de riesgo, resulta interesante la evaluación a través de la pérdida de resistencia mecánica.

La impregnación de una madera con productos a base de cobre, cromo, arsénico (CCA), mediante procesos de vacío-presión, prolonga la vida útil de dicha madera en servicio, por más de 30 años. Otras especies, como la acacia blanca "Robinia pseudoacacia L.", presentan una adecuada durabilidad natural para estos usos sin necesidad de aplicación de dicho tratamiento preservante.

El objetivo de este trabajo fue determinar y evaluar, el contenido de humedad, la densidad aparente normal y la resistencia mecánica en flexión estática, en madera de álamo criollo impregnada con CCA por vacío presión (método Bethell), y compararla con la misma madera sin impregnar y con acacia blanca de reconocida durabilidad natural.

Se determinó contenido de humedad (IRAM 9532), densidad aparente normal (IRAM 9544) y módulo de rotura (MOR) en flexión estática (IRAM 9545). El material (probetas) provino de tres cementerios de estacas ubicados en Río Gallegos, Gobernador Gregores y Perito Moreno (Santa Cruz, Argentina).

\footnotetext{
1 Ingeniero Forestal. Facultad de Ciencias Agrarias y Forestales. Universidad Nacional de La Plata (UNLP) 60 y 119. CP: 1900. CC 31-La Plata. Argentina. gabrieldkeil@yahoo.com.ar

2 Ingeniero Forestal

3 Ingeniero Forestal. Facultad de Ciencias Agrarias y Forestales. Universidad Nacional de La Plata (UNLP) 60 y 119. CP: 1900. CC 31-La Plata. Argentina.

4 Ingeniero Forestal

5 Facultad de Ciencias Agrarias y Forestales. UNLP. 60 y 119. CP: 1900. CC 31-La Plata. Argentina.
} 
Del análisis de los resultados surge que la madera de álamo criollo impregnada con CCA, mediante el método Bethell, y expuesta al exterior en contacto con el suelo (categoría de riesgo R4, IRAM 9600), durante 4 años, mantuvo sus características resistentes, al igual que la madera de acacia blanca expuesta a las mismas condiciones. Además, dichos resultados no fueron significativamente influenciados por los sitios de exposición.

Palabras clave: Patagonia, impregnación, CCA, cementerios, densidad, flexión estática

\section{SUMMARY}

In the Patagonia in general and in the Santa Cruz province in particular, there is an important resource of Populus nigra cv Italica, mainly in protective curtains to vegetable and fruit crops which could be a regional important wood resource. Among the virtues of the wood of this species are its light color, right grain, the absence of extractives and, in spite of its low natural durability, can be easily preserved.

The degradation field trials allow assessing the natural or acquired durability of wood exposed to different risk conditions similar to those of the wood in service. The traditional evaluation of these tests is through the wood weight loss, however it is interesting to make also a quantitative assessment of the mechanical strength loss.

The impregnation of wood with copper, chromium and arsenic (CCA) based products, using vacuum-pressure process, extend the life of wood in service for over 30 years. Other species, such as Robinia pseudoacacia L., have a good natural durability without preservation treatments.

The objective of this study was to determine and assess, moisture content, normal apparent density and mechanical strength in static bending in Populus nigra cv Italica wood impregnated with CCA through of vacuum pressure (Bethell method), and to compare it to untreated wood of the same species and of Robinia pseudoacacia.

Moisture content: CH (IRAM 9532): Dn (IRAM 9544) and rupture modulus (MOR) in static bending (IRAM 9545) were determined. The material came from three stakes cemeteries located in Rio Gallegos, Gobernador Gregores and Perito Moreno, Santa Cruz, Argentina.

According to the results, it appears that Populus nigra cv Italica wood impregnated with CCA, through Bethell method, and exposed to the outside contact with the ground (R3risk category, IRAM 9600), for four years, maintains strength characteristics similar to those of Robinia pseudoacacia wood exposed to the same conditions. The results were not significantly influenced by exposure sites.

Key words: Patagonia, impregnation, CCA, cemeteries, density, static bending 


\section{INTRODUCCION}

En la República Argentina existen aproximadamente 64.000 ha forestadas con especies del género Populus (álamos), siendo las principales zonas productoras el Delta del Paraná, Cuyo, Patagonia y la Pampa Húmeda (Achinelli, 2006).

En la región patagónica en general y en la provincia de Santa Cruz en particular, existe un recurso importante de álamo criollo, Populus nigra cv Italica, concentrado principalmente en cortinas protectoras de cultivos fruti-hortícolas (Peri y Martinez Pastur, 1998), lo que podría constituir un importante recurso maderero regional.

Dentro de las virtudes de la madera de esta especie, se encuentran su color claro, su grano derecho, la ausencia de extractivos y, si bien su durabilidad natural es baja y limita su uso en condiciones de riesgo severas, presenta la característica de ser fácilmente impregnable (IRAM 9600, 1998).

Históricamente, dicha madera ha sido utilizada en la región, principalmente con destinos rurales (poste corto y leña). Sin embargo, nunca ha existido un aprovechamiento intensivo de ella, debido fundamentalmente a su baja durabilidad natural, inferior a 5 años (IRAM 9600, 1998).

Asimismo en dicha región, existe un recurso limitado de acacia blanca Robinia pseudoacacia $\mathrm{L}$, con excelente durabilidad natural, lo cual hace que dicha madera sea utilizada para productos de carpintería rural.

El uso de maderas no durables o poco durables pero tratadas a presión con algún producto preservante prolonga la vida útil en servicio por más de 30 años (JUNAC, 1988; IRAM 9600, 1998).

Entre los productos disponibles en el mercado, el arseniato de cobre cromatado; CCA (cromo, cobre, arsénico), ha presentado buenos resultados y múltiples aplicaciones de diferentes maderas han podido beneficiarse con sus bondades. Dentro de dichas aplicaciones pueden mencionarse viñas, parrales, madera para construcción, postes de transmisión eléctrica y teléfonos, cercos agrícolas y ornamentales, juegos infantiles, muebles rústicos y terrazas en exteriores entre otras aplicaciones (IRAM 9600, 1998).

La impregnación es un proceso industrial que permite introducir algún producto preservante en la madera, prolongando de este modo su vida útil, haciendo frente a una amplia variedad de situaciones ambientales para las cuales no presenta una durabilidad natural significativa (JUNAC, 1988).

En este sentido, debido a que se cuenta con un recurso importante de álamo criollo en la región, sería interesante plantear la impregnación de dicha madera con CCA para aumentar su vida útil en servicio, y de esta manera poder complementar o reemplazar, en los usos que así lo permitan, a la madera de acacia blanca destinando esta última a la fabricación de productos de mayor valor agregado. 
Los ensayos de degradación a campo (cementerios de estacas), constituyen una herramienta para evaluar la durabilidad natural o adquirida de la madera expuesta a distintas condiciones edafoclimáticas y biológicas (JUNAC, 1988). Asimismo, el nivel de retención del preservante necesario para asegurar una vida útil prolongada de la madera, se determina de acuerdo con las condiciones a las que se expone la madera al ataque de agentes destructores (IRAM 9600, 1998).

Como hipótesis de trabajo se consideró que impregnando la madera de álamo criollo con CCA por el método industrial de Bethell, ésta podría tener prestaciones similares a las de acacia blanca, lo cual permitiría reemplazarla para determinados usos y aplicaciones estructurales considerando el dimensionamiento de las piezas.

\section{OBJETIVO}

El objetivo de este trabajo fue determinar y evaluar, el contenido de humedad, la densidad aparente normal y la resistencia mecánica en flexión estática, en madera de álamo criollo impregnada con CCA por vacío presión (método Bethell), y compararla con la misma madera sin impregnar y con acacia blanca de reconocida durabilidad natural.

\section{MATERIAL Y MÉTODO}

\section{Material Leñoso}

Se trabajó con probetas de madera $(20 \mathrm{~mm} \times 20 \mathrm{~mm} \times 300 \mathrm{~mm})$ de álamo criollo sin impregnar (testigo) e impregnada y con madera de acacia blanca. Las maderas fueron impregnadas por el método de vacío presión en autoclave a célula llena (Bethell) y el preservante utilizado fue arseniato de cobre cromatado (CCA) con retenciones establecidas en la norma IRAM 9600, 1998 para latifoliadas y categoria de riesgo R4 (al exterior en contacto con el suelo).

Luego de la preservación, la madera se estivó en un lugar protegido de las condiciones ambientales y a la sombra para favorecer la difusión del CCA, durante 15-20 días. Para evidenciar la penetración del preservante se pincelaron las escuadrías con cromo azurol S.

El material leñoso estuvo enterrado hasta el 50\% de su longitud durante 4 años en los sitios y condiciones detallados en el Cuadro $\mathrm{N}^{\circ} 1$. 


\section{Cuadro $\mathrm{N}^{\circ} 1$ \\ SITIOS DE EXPOSICIÓN}

\begin{tabular}{|c|c|c|c|}
\hline SITIOS & PERITO MORENO & $\begin{array}{l}\text { GOBERNADOR } \\
\text { GREGORES }\end{array}$ & RIO GALLEGOS \\
\hline Coordenadas & $\begin{array}{l}46^{\circ} 51^{\prime}-47^{\circ} 13^{\prime} \text { LS } \\
71^{\circ} 11^{\prime}-70^{\circ} 19^{\prime} \text { LO }\end{array}$ & $\begin{array}{l}48^{\circ} 40^{\prime}-49^{\circ} 12^{\prime} . \text { LS } \\
69^{\circ} 08^{\prime}-70^{\circ} 20^{\prime} \text { LO }\end{array}$ & $\begin{array}{l}51^{\circ} 22^{\prime}-52^{\circ} 00^{\prime} \text { LS } \\
72^{\circ} 26^{\prime}-69^{\circ} 12^{\prime} \text { LO }\end{array}$ \\
\hline Región Natural & Patagonia Extra-andina & Patagonia. Extra-andina & $\begin{array}{c}\text { Patagonia. Extra-andina } \\
\text { Austral }\end{array}$ \\
\hline Ordenes de Suelos & $\begin{array}{l}\text { En su mayoría } \\
\text { entisoles }\end{array}$ & En su mayoría aridisoles & $\begin{array}{c}\text { En su mayoría aridisoles y } \\
\text { molisoles. }\end{array}$ \\
\hline Tipo Climático & Templado frío árido & Templado frío árido & $\begin{array}{l}\text { Templado frío, subhúmedo } \\
\text { en el oeste a semiárido en } \\
\text { el este. }\end{array}$ \\
\hline $\begin{array}{l}\text { Precipitación Media } \\
\text { Anual }(\mathrm{mm})\end{array}$ & 72,9 & 191,7 & $\begin{array}{c}260 \\
\begin{array}{c}\text { (estación más lluviosa en } \\
\text { otoño) }\end{array} \\
\end{array}$ \\
\hline $\begin{array}{l}\text { Temperatura Media } \\
\text { Anual }\left({ }^{\circ} \mathrm{C}\right)\end{array}$ & 7,8 & 8,5 & 6,9 \\
\hline $\begin{array}{l}\text { Velocidad Media del } \\
\text { Viento }(\mathrm{Km} / \mathrm{h})\end{array}$ & 35 & 45 & 20 \\
\hline
\end{tabular}

(Fuente: Secretaria de Minería de la Nación y Servicio Meteorológico Nacional)

Transcurridos los 4 años de exposición, el material fue extraído de los cementerios de estacas, limpiado con pincel y acondicionado para su envío a la ciudad de La Plata, donde se realizaron los estudios tecnológicos. Dichos estudios fueron realizados en el laboratorio de Industrias de Transformación Mecánica de la Facultad de Ciencias Agrarias y Forestales de la UNLP, previo acondicionado (durante 30 días), hasta alcanzar el contenido de humedad de equilibrio higroscópico, testeado periódicamente mediante higrómetro electrónico GANN, Hydromette HT 85.

\section{Ensayo Mecánico}

El ensayo mecánico realizado fue el de flexión estática (IRAM 9545, 1985). El mismo fue realizado en el Laboratorio de Ensayos de Materiales, Facultad de Ingeniería, UNLP, en prensa universal de $5000 \mathrm{Kg}$ Alfred J. Amsler y Co, Schaffouse - Suisse 7928.

Se trabajó con un total de 270 probetas, 30 probetas por tratamiento. Con los datos obtenidos se calculó el módulo de rotura (MOR) en flexión estática.

\section{Propiedades Físicas}

Las propiedades físicas estudiadas fueron contenido de humedad CH (IRAM 9532, 1963) y densidad aparente normal Dn (IRAM 9544, 1985). En este caso las probetas utilizadas 
en el ensayo mecánico fueron nuevamente procesadas en carpintería para la obtención de 330 probetas cúbicas de $20 \mathrm{~mm}$ de lado.

Para la determinación del $\mathrm{CH}$ se utilizó el método de secado en estufa. Se utilizó balanza analítica con una precisión de $10 \mathrm{Mg}$, estufa de secado con termómetro y termostato regulable a $103^{\circ} \mathrm{C} \pm 2^{\circ} \mathrm{C}$ y desecador, provisto de cloruro de calcio como deshidratante.

Para la determinación de la densidad aparente normal a cada una de las probetas se le determinó el peso húmedo inicial $(\mathrm{Ph})$ con balanza de precisión y luego, por el método de desplazamiento de fluidos, se determinó su volumen húmedo inicial (Vh). Los ensayos se llevaron a cabo en laboratorios del INFIVE, Xilotecnología e Industria de Transformación Mecánica, Departamento de Ingeniería Agrícola y Forestal, Facultad de Ciencias Agrarias y Forestales, UNLP.

\section{Análisis Estadístico} variabilidad.

Al conjunto de datos de cada variable se le calculó la media y el coeficiente de

Los datos obtenidos para todas las especies fueron estudiados a partir del Análisis de la Varianza y ante diferencias significativas $(p<0,05)$ se aplicó el test de comparación de medias.

\section{RESULTADOS Y DISCUSION}

\section{Propiedades Físicas} de exposición.

En el Cuadro $\mathrm{N}^{\circ} 2$ se presentan los valores de $\mathrm{CH}$ y Dn de las especies en los tres sitios

Cuadro $\mathrm{N}^{\circ} 2$

CH Y DN EN LOS TRES SITIOS DE ESTUDIO

\begin{tabular}{|c|c|c|c|c|c|c|}
\hline \multirow[b]{2}{*}{ Especie } & \multicolumn{2}{|c|}{ Río Gallegos } & \multicolumn{2}{|c|}{ Gobernador Gregores } & \multicolumn{2}{|c|}{ Perito Moreno } \\
\hline & $\begin{array}{l}\mathrm{CH} \\
\text { (\%) }\end{array}$ & $\begin{array}{c}\mathrm{Dn} \\
(\mathrm{Kg} / \mathrm{m} 3)\end{array}$ & $\begin{array}{l}\mathrm{CH} \\
(\%)\end{array}$ & $\begin{array}{c}\text { Dn } \\
(\mathrm{Kg} / \mathrm{m} 3)\end{array}$ & $\begin{array}{l}\mathrm{CH} \\
(\%)\end{array}$ & $\begin{array}{c}\mathrm{Dn} \\
(\mathrm{Kg} / \mathrm{m} 3)\end{array}$ \\
\hline Álamo Testigo & $\begin{array}{l}9,63 \mathbf{a} \\
(1,54)\end{array}$ & $\begin{array}{l}330 \mathbf{a} \\
11,16\end{array}$ & $\begin{array}{l}9,22 \mathbf{a} \\
(9,03)\end{array}$ & $\begin{array}{c}330 \mathbf{a} \\
(11,17)\end{array}$ & $\begin{array}{c}9,49 \mathbf{a} \\
(13,60)\end{array}$ & $\begin{array}{c}370 \mathbf{a} \\
(10,62)\end{array}$ \\
\hline Álamo con CCA & $\begin{array}{c}9,26 \mathbf{a} \\
(13,57)\end{array}$ & $\begin{array}{l}340 \mathbf{a} \\
(1,57)\end{array}$ & $\begin{array}{l}8,41 \mathbf{a} \\
(5,96)\end{array}$ & $\begin{array}{c}390 \mathrm{a} \\
(11,74)\end{array}$ & $\begin{array}{c}9,69 \mathbf{a} \\
(17,87)\end{array}$ & $\begin{array}{l}370 \mathbf{a} \\
(9,12)\end{array}$ \\
\hline Acacia blanca & $\begin{array}{l}7,70 \mathbf{a} \\
(1,83)\end{array}$ & $\begin{array}{l}820 \mathbf{b} \\
(2,54)\end{array}$ & $\begin{array}{c}8,78 \mathbf{a} \\
(12,88)\end{array}$ & $\begin{array}{l}780 \mathbf{b} \\
(6,14)\end{array}$ & $\begin{array}{l}8,93 \mathbf{a} \\
(5,18)\end{array}$ & $\begin{array}{l}810 \mathbf{b} \\
(2,56)\end{array}$ \\
\hline
\end{tabular}

Diferentes letras denotan diferencias significativas con el test de Tukey $(p<0.05)$.

Dn densidad aparente normal; $\mathrm{CH}$ contenido de humedad.

El coeficiente de variabilidad se indica entre paréntesis. 
El CH influye en la determinación de las propiedades físicas y mecánicas ya que modifica el volumen y la masa del material, (Coronel, 1994).

Según se observa en el Cuadro $\mathrm{N}^{\circ} 2$, el $\mathrm{CH}$ determinado no presentó diferencias significativas entre especies en los tres sitios de estudio.

La Dn es importante debido a que es la densidad más estable de la madera en uso y en consecuencia la que se toma como referencia para las determinaciones de resistencia mecánica (Coronel, 1994). Su valor se tiene en cuenta en la industria de la madera aserrada y en productos de madera sólida.

Los valores de Dn determinados no se diferenciaron entre la madera de álamo testigo y la madera de álamo impregnado, pero si hubo diferencias significativas en ambos casos, con la madera de acacia blanca. Dicho comportamiento se presentó en los tres sitios de estudio.

En los tres sitios, la Dn de la madera de álamo testigo y de álamo con CCA fue baja (madera liviana), mientras que la densidad de la acacia blanca fue alta (madera densa o pesada) (Rivero Moreno, 2004).

\section{Cuadro $\mathrm{N}^{\circ} 3$ \\ VALORES DE CH COMPARADOS ENTRE SITIOS}

\begin{tabular}{|l|c|c|c|c|c|c|}
\hline \multirow{2}{*}{ Sitio } & \multicolumn{2}{|c|}{ Álamo Testigo } & \multicolumn{2}{c|}{ Álamo con CCA } & \multicolumn{2}{c|}{ Acacia Blanca } \\
\cline { 2 - 7 } & $\begin{array}{c}\text { CH } \\
(\%)\end{array}$ & $\begin{array}{c}\text { Dn } \\
(\mathbf{K g} / \mathbf{m} 3)\end{array}$ & $\begin{array}{c}\text { CH } \\
(\%)\end{array}$ & $\begin{array}{c}\text { Dn } \\
(\mathbf{K g} / \mathbf{m} 3)\end{array}$ & $\begin{array}{c}\mathbf{C H} \\
(\%)\end{array}$ & $\begin{array}{c}\text { Dn } \\
(\mathbf{K g} / \mathbf{m} 3)\end{array}$ \\
\hline \multirow{2}{*}{ Gobernador Gregores } & $9,22 \mathbf{a}$ & $330 \mathbf{a}$ & $8,41 \mathbf{a}$ & $390 \mathbf{a}$ & $8,78 \mathbf{a}$ & $780 \mathbf{a}$ \\
& $(9,03)$ & 11,17 & $(5,96)$ & $(11,74)$ & $(12,88)$ & $(6,14)$ \\
\hline \multirow{2}{*}{ Perito Moreno } & $9,49 \mathbf{a}$ & $370 \mathbf{a}$ & $9,69 \mathbf{a}$ & $370 \mathbf{a}$ & $8,93 \mathbf{a}$ & $810 \mathbf{a}$ \\
& $(13,60)$ & $(10,62)$ & $(17,87)$ & $(9,12)$ & $(5,18)$ & $(2,56)$ \\
\hline \multirow{2}{*}{ Río Gallegos } & $9,63 \mathbf{a}$ & $330 \mathbf{a}$ & $9,26 \mathbf{a}$ & $340 \mathbf{a}$ & $7,70 \mathbf{a}$ & $820 \mathbf{a}$ \\
& $(1,54)$ & $(11,16)$ & $(13,57)$ & $(1,57)$ & $(1,83)$ & $(2,54)$ \\
\hline
\end{tabular}

Diferentes letras denotan diferencias significativas con el test de Tukey $(p<0.05)$.

Los coeficientes de variación (CV\%) se indican entre paréntesis.

Al realizar el test de comparación de medias de las variables $\mathrm{CH}$ y $\mathrm{Dn}$ en las tres localidades (Cuadro $\mathrm{N}^{\circ} 3$ ) se observó que los valores no difirieron significativamente entre los sitios.

\section{Propiedades Mecánicas} de exposición.

En el Cuadro $\mathrm{N}^{\circ} 4$ se presentan los valores de MOR de las especies en los tres sitios

Del Cuadro $\mathrm{N}^{\circ} 4$ se desprende que la madera de álamo testigo e impregnado se diferenció de la madera de acacia blanca en los tres sitios de estudio. Asimismo, el testigo 
se diferenció del álamo impregnado en el sitio de Gobernador Gregores mientras que no se diferenció en los otros dos sitios.

En todos los casos, la madera de álamo testigo y álamo impregnado presentaron valores muy bajos de resistencia a la rotura en flexión estática, mientras que la madera de acacia blanca fue altamente resistente a dicho esfuerzo en los tres sitios de exposición (Rivero Moreno, 2004).

\section{Cuadro $\mathrm{N}^{\circ} 4$ \\ RESULTADOS DE FLEXIÓN ESTÁTICA EN LOS TRES SITIOS DE ESTUDIO}

\begin{tabular}{|l|c|c|c|}
\hline \multirow{3}{*}{ Especie } & Río Gallegos & Gobernador Gregores & Perito Moreno \\
\cline { 2 - 4 } & $\begin{array}{c}\text { MOR } \\
(\mathbf{N} / \mathbf{m m} 2)\end{array}$ & $\begin{array}{c}\text { MOR } \\
(\mathbf{N} / \mathbf{m m} 2)\end{array}$ & $\begin{array}{c}\text { MOR } \\
(\mathbf{N} / \mathbf{m m} 2)\end{array}$ \\
\hline \multirow{2}{*}{ Álamo Testigo } & $27,32 \mathbf{a}$ & $19,50 \mathbf{a}$ & $36,30 \mathbf{a}$ \\
& $(11,71)$ & $(20,18)$ & $(42,10)$ \\
\hline \multirow{2}{*}{ Álamo con CCA } & $32,90 \mathbf{a}$ & $45,70 \mathbf{b}$ & $33,88 \mathbf{a}$ \\
& $(15,5)$ & $(19,13)$ & $(29,54)$ \\
\hline \multirow{2}{*}{ Acacia blanca } & $142,00 \mathbf{b}$ & $137,00 \mathbf{c}$ & $161,30 \mathbf{b}$ \\
& $(14,86)$ & $(17,32)$ & $(12,32)$ \\
\hline
\end{tabular}

Diferentes letras denotan diferencias significativas con el test de Tukey $(p<0.05)$.

Los coeficientes de variación (CV\%) se indican entre paréntesis

Los resultados obtenidos para el álamo testigo e impregnado no concuerdan con lo planteado por Suirezs (2005) para la madera de pino taeda (Pinus taeda), respecto de que la madera impregnada con CCA ofrece menor resistencia al módulo de rotura perdiendo tenacidad.

Al realizar el test de comparación de medias de la variable MOR en las tres localidades (Cuadro $N^{\circ} 5$ ), se observó que los MOR del álamo impregnado y de la acacia blanca en el sitio de Gobernador Gregores se diferenciaron con respecto a los otros sitios, no diferenciándose entre sitios el álamo testigo.

\section{Cuadro $\mathrm{N}^{\circ} 5$}

\section{RESULTADOS DEL ENSAYO DE FLEXIÓN ESTÁTICA COMPARADOS ENTRE SITIOS}

\begin{tabular}{|l|c|c|c|}
\hline \multirow{2}{*}{ Sitio } & Álamo Testigo & Álamo con CCA & Acacia Blanca \\
\cline { 2 - 4 } & \multicolumn{3}{|c|}{ MOR (N/mm2) } \\
\hline \multirow{2}{*}{ Gobernador Gregores } & $19,50 \mathbf{a}$ & $45,70 \mathbf{a}$ & $137,00 \mathbf{a}$ \\
& $(20,18)$ & $(19,13)$ & $(17,32)$ \\
Perito Moreno & $36,30 \mathbf{a}$ & $33,88 \mathbf{b}$ & $(161,30 \mathbf{b}$ \\
& $(42,10)$ & $(29,54)$ & $12,32)$ \\
Río Gallegos & $27,32 \mathbf{a}$ & $32,90 \mathbf{b}$ & $(14,00 \mathbf{b}$ \\
\hline
\end{tabular}

Diferentes letras denotan diferencias significativas con el test de Tukey $(p<0.05)$.

Los coeficientes de variación (CV\%) se indican entre paréntesis 


\section{CONCLUSIONES}

Según las propiedades tecnológicas estudiadasl la madera de álamo criollo impregnada con CCA ofrece prestaciones similares a las de madera en estado original, sin reducción de la resistencia a flexión pero con valores de MOR inferiores al de acacia blanca, situación a considerar en el dimensionado de las piezas de madera.

En general, las condiciones edafoclimáticas del sitio de exposición no influyeron sobre los parámetros tecnológicos estudiados.

En base a los resultados obtenidos, es posible la complementación o el reemplazo de la madera de acacia blanca por madera de álamo impregnada con CCA en usos de carpintería rural para situaciones de riesgo similares.

\section{REFERENCIAS}

Achinelli, F. G., 2006. Silvicultura de álamos y sauces en la pampa húmeda. Actas Jornadas de Salicáceas 2006. $16 \mathrm{pp}$

Coronel, E. O., 1994. Fundamentos de las propiedades físicas y mecánicas de la madera. Aspectos teóricos y prácticos para la determinación de las propiedades y sus aplicaciones. 1 Parte: Fundamentos de las propiedades físicas de la madera. Publicación ITM - UNSE. $187 \mathrm{pp}$.

IRAM 9532, 1963. Método de determinación de humedad. Instituto de Racionalización de Materiales. $14 \mathrm{pp}$.

IRAM 9544, 1985. Método para la determinación de la densidad aparente. Instituto Argentino de Racionalización de Materiales. 10 pp.

IRAM 9545. 1985. Maderas: Método de ensayo de flexión estática. Instituto Argentino de Racionalización de materiales $10 \mathrm{pp}$.

IRAM 9600, 1998. Preservación de maderas: Maderas preservadas mediante procesos con presión en autoclave. Instituto Argentino de Racionalización de Materiales. 23 pp.

JUNAC, 1988. Junta del Acuerdo de Cartagena. Manual del Grupo Andino para la preservación de Maderas. 1 Ed., Lima. Perú. Pp 294.

Peri, P. y Martinez Pastur, G., 1998. Crecimiento en cortinas cortaviento de Populus nigra cv 'Italica' en Patagonia Sur - Argentina. Investigación Agraria: Sistemas y Recursos Forestales 7(1-2): 73-83.

Rivero Moreno, J., 2004. Propiedades Físico-Mecánicas de Gmelina arborea Roxb. y Tectona grandis Linn. F. Proveniente de Plantaciones Experimentales del Valle del Sacta-Cochabamba. Cochabamba. Bolivia. http://www.monografias.com. 73 pp. 
Suirezs, T., 2000. Efecto de la impregnación con CCA (cromo-cobre-arsénico) sobre las propiedades físicas y mecánicas de la madera de Pinus taeda implantado en la provincia de Misiones. Tesis Universidad Nacional de Misiones.76 pp. 